\title{
A PROSPECTIVE ASSESSMENT OF POLYPHARMACY INDUCED DRUG INTERACTIONS WITH CORTICOSTEROIDS \\ S Kumar ${ }^{1 *}$, PK Thakur², KK Jha'2, SK Shah ${ }^{2}$
}

\author{
${ }^{1}$ Department of Pharmacology, N.E.T. Pharmacy College, Raichur, Karnataka, India \\ ${ }^{2}$ Department of Pharmacy Practice, N.E.T. Pharmacy College, Raichur, Karnataka, India \\ *Correspondence to: Dr Shiv Kumar, Department of Pharmacology, N.E.T. Pharmacy College, Raichur- 584103, Karnataka, India. \\ Email: shivkumarmatur@gmail.com.
}

\begin{abstract}
Drug interaction represents a major problem in day-to-day practice. The incidence of adverse reactions increases almost exponentially as the number of drugs co prescribed rises, and this is in part due to interaction. This study was aimed to study the incidence of polypharmacy induced drug interactions with corticosteroids and their severity. A prospective observational study was carried out in 211 In-patients from various departments of the hospital during 6 months and subjected to statistical analysis. Out of 211 In-patients, 142 (67.3\%) were male and 69 (32.7\%) were female, A with maximum (44.9\%) belonging to geriatric age group. Most of patients were prescribed with more than 6 drugs. The numbers of prescriptions having drug interactions with corticosteroids were 111 , with majority (49.5\%) of them were from general medicine ward. A total of 154 drug-drug interactions were found among these prescriptions including 124 moderate, 21 major and 9 contraindicated interactions. Most of the prescriptions (75) were having atleast 1 interaction and 28 prescriptions were having 2 interactions. Out of these interactions, 17, 75, and 62 were having excellent, good and fair scientific evidence. Regarding the onset of these drug-drug interactions, 74(48\%) were with delayed onset, $43(27.9 \%)$ were unspecified and $37(24 \%)$ were with rapid onset. Dexamethasone with ciprofloxacin, tramadol, moxifloxacin, diclofenac, pantoprazole, theophylline were the most encountered combinations. A high prevalence of polypharmacy and drug interactions with corticosteroids was identified in our study. Since concurrent drugs can potentiate irreversible adverse effects of corticosteroids, a regular therapeutic intervention is necessary.
\end{abstract}

Key words: Corticosteroids, Drug Interactions, Polypharmacy.

\section{INTRODUCTION}

Drug interaction represents a major problem in dayto-day practice. The incidence of adverse reactions increases almost exponentially as the number of drugs co prescribed rises, and this is in part due to interaction. ${ }^{1}$

Appropriate drug utilization is beneficial in global reduction of morbidity and mortality with its consequent medical, social and economic benefits.

Drugs are the most common medical interventions for betterment of patients but it had recognized long ago that they are fatal too. The saying rightly goes about the drugs that "Drugs are Double Edged Weapons".

Critically ill, chronically ill and elderly patients are particularly at risk of drug interactions due to polypharmacy as well as impaired homeostatic mechanisms ${ }^{4}$. It is reported in several studies that elder patients (61-80 years) had more prevalence of polypharmacy and drug-drug interactions compared to the other age groups. The use of multiple medications increases the possibility of drug interactions and adverse reactions to drugs, poorer compliance, increased risk of hospitalization and medical errors caused by drugs.

The definition of polypharmacy in the literature is not uniform. However, the word 'poly' is a Greek word and means many or much. ${ }^{6}$ There are basically two approaches to the definition. The first refers only to the number of drugs taken 
simultaneously. According to this, polypharmacy means the concurrent use of 2 or more drugs. However, some authors distinguish between minor polypharmacy (the concurrent use of 3 to 5 drugs) and major polypharmacy (the concurrent use of 5 or more drugs). ${ }^{7,8}$ The other approach to the definition focuses on the clinical indication and the effect of the administered medication. According to this definition, irrational, clinically not indicated drug use is regarded as polypharmacy. ${ }^{9}$

Corticosteroids are adrenal steroids, indicated for suppression of inflammation, suppression of immune response and replacement therapy. These life saving drugs play a major role in treatment of chronic diseases like asthma, COPD, arthritis and other diseases affecting skin, GIT and CNS. Use of these drugs must be carefully weighed in each patient since they produces number of complications. The goal of corticosteroids therapy is to use the safest and least number of drugs to get more efficacy in short period and to avoid ADRs \& drug-drug interactions. Keeping present scenario in mind, this study was aimed to assess the incidence of polypharmacy induced drug interactions with corticosteroids with their severity and to identify whether it is associated with Polypharmacy.

\section{MATERIALS \& METHODS}

\section{Study Design:}

A prospective observational study

\section{Study Site:}

The study was conducted in the various departments of Navodaya Medical College Hospital and Research centre, Raichur, Karnataka, after obtaining the ethical clearance.

\section{Study Period:}

The study was conducted for 6 months period during November 2014 to April 2015.

\section{Study Population:}

A total of 211 patients were included in the study.

\section{Study Criteria:}

Inclusion criteria:
In-patients prescribed with corticosteroids from General Medicine, Orthopedic, ICU \& Emergency, Pediatrics and Pulmonary Medicine.

\section{Exclusion criteria:}

- The patients from departments other than mentioned in inclusion criteria.

- Pregnancy and lactating women

\section{Sources of Data:}

The data was collected from various sources such as patient's case reports, laboratory data, treatment charts and patient interview/patient care taker interview using specially designed data collection form.

\section{Study protocol:}

Patient who met the study criteria were included in the study. Demographics, lab data, diagnosis and treatment chart were noted. All the cases were reviewed prospectively and monitored extensively, the pattern of corticosteroids uses like their category, indication, and rationality of the prescription, concurrent drugs prescribed and/or number of drugs in prescriptions. Drug-drug interactions were identified and documented by using MICROMEDEX $2.0^{\circ}$ software, drug.com and STOCKLEY's book. Potential drug-drug interactions were categorized into different levels as follows. ${ }^{11}$

\section{Onset}

- Rapid: The effect of interaction occurs within 24 hours of administration.

- Delayed: The effect occurs if the interacting combination is administered for more than 24 hours, i.e., days to week(s).

- Unspecified: The occurrence of effect of interaction is not specified.

\section{Severity}

- Contraindicated: The drug-combination is contraindicated for concurrent use.

- Major: There is risk of death and/or medical intervention is required to prevent or minimize serious negative outcomes.

- Moderate: The effect of interaction can 
deteriorate patient's condition and may require alteration of therapy.

- Minor: Little effects are produced that don't impair therapeutic outcome and there is no need of any major change in therapy.

\section{Scientific evidence (Documentation)}

- Excellent: The interaction has been clearly demonstrated in well-controlled studies.

- Good: Studies strongly suggest that the interaction exists except proof of well-controlled studies.

- Fair: Available evidences are poor, but the interaction is suspected on the basis of pharmacologic considerations; or, evidences are good for an interaction of pharmacologically similar drug.

- Poor: Theoretically the interaction may occur but reports are very limited, such as few case report.

\section{Statistical analysis:}

Data was analyzed using descriptive statistics namely total numbers, percentage, mean and standard deviation wherever applicable.

\section{RESULTS AND DISCUSSION}

\section{Patient Demographics}

As per patient demographic data obtained, out of 211 In-patients, 142(67.3\%) were male and 69(32.7\%) were female. A maximum of $48(22.7 \%)$ patients belonged to age group of 51-60 years followed by $47(21.8 \%)$ patients from the age group of more than 60 years. This data showed that commonly geriatrics male populations are more prone to diseases, for which corticosteroids are prescribed. (Table 1 )

Table 1: Gender and Age Wise Distribution

\begin{tabular}{|c|c|c|c|c|c|c|}
\hline Age group (years) & Male & $\begin{array}{c}\text { Percentage } \\
\text { (\%) }\end{array}$ & Female & $\begin{array}{c}\text { Percentage } \\
\text { (\%) }\end{array}$ & Total & $\begin{array}{c}\text { Percentage } \\
\text { (\%) }\end{array}$ \\
\hline $0-10$ & 23 & 10.9 & 12 & 5.7 & 35 & 16.5 \\
\hline $11-20$ & 6 & 2.8 & 6 & 2.8 & 12 & 5.6 \\
\hline $21-30$ & 8 & 3.7 & 5 & 2.3 & 13 & 6.1 \\
\hline $31-40$ & 15 & 7.1 & 9 & 4.3 & 24 & 11.3 \\
\hline $41-50$ & 23 & 10.9 & 9 & 4.3 & 32 & 15.1 \\
\hline $51-60$ & 31 & 14.6 & 17 & 8.0 & 48 & 22.7 \\
\hline$>60$ & 36 & 17.1 & 11 & 5.2 & 47 & 22.2 \\
\hline Total & 142 & 67.3 & 69 & 32.7 & 211 & 100 \\
\hline
\end{tabular}

Incidence of Polypharmacy

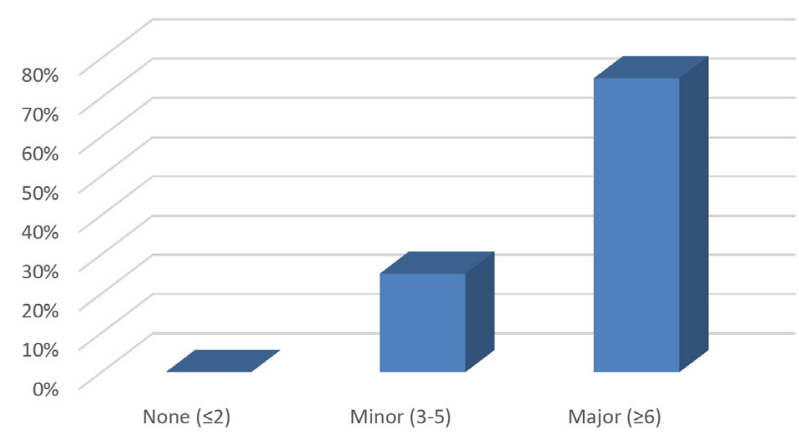

Class of Polypharmacy

Figure 1: Incidence of Polypharmacy

\section{Drug-Drug interaction Observed}

Table 2 showed ward wise distribution and prescriptions containing corticosteroids interactions along with their severity. Out of 211 In-patients, 111 prescriptions were found to have drugdrug interactions with corticosteroids. Majority 94(44.5\%) were from General Medicine ward followed by padiatric ward $37(17.5 \%)$. Out of these 111 prescriptions, a total of 154 interactions were found. Most of the prescriptions (75) were having atleast 1 interaction and 28 prescriptions were having 2 interactions. 
This data confirms that patients admitted in General Medicine ward have higher exposure to polypharmacy including corticosteroids and drug-drug interaction. (Table 2)

Table 2: Drug-Drug Interaction Observed with corticosteroids

\begin{tabular}{|l|l|l|l|l|l|}
\hline Ward & $\begin{array}{l}\text { No. of } \\
\text { Patients, (\%) }\end{array}$ & $\begin{array}{l}\text { No. of prescriptions } \\
\text { with Corticosteroids } \\
\text { interaction, (\%) }\end{array}$ & $\begin{array}{l}\text { No. of moderate } \\
\text { interaction }\end{array}$ & $\begin{array}{l}\text { No. of major } \\
\text { interaction }\end{array}$ & $\begin{array}{l}\text { No. of contraindicated } \\
\text { interaction }\end{array}$ \\
\hline General med. & $94(44.5)$ & $55(49.5)$ & 66 & 17 & 7 \\
\hline Pulmonary med. & $31(14.7)$ & $13(11.7)$ & 14 & 0 & 0 \\
\hline Paediatrics & $37(17.5)$ & $15(13.5)$ & 15 & 1 & 1 \\
\hline Orthopaedics & $16(7.6)$ & $12(10.8)$ & 10 & 1 & 0 \\
\hline Emerg. \& causality & $21(9.9)$ & $8(7.2)$ & 9 & 2 & 1 \\
\hline ICU & $12(5.6)$ & $8(7.2)$ & 10 & 0 & 0 \\
\hline Total & $211(100)$ & $111(52.6)$ & 124 & 21 & 9 \\
\hline
\end{tabular}

\section{Prevalence of Drug-Drug Interactions}

One hundred fifty four $(72.9 \%)$ patients had at least one potential to drug interaction if prescribed with corticosteroids regardless of type of severity, $124(80.5 \%)$ moderate, $21(13.6 \%)$ major and $9(5.8 \%)$ contraindicated interactions.

In around $68 \%$ of patients were identified with one interaction followed by $25.3 \%$ of two, $5.1 \%$ of three and $1.9 \%$ of four interactions. This discrepancy in prevalence may be result of high utilization of drugs having more interacting potentials in our setup. This confirms that higher prevalence of drugdrug interactions associated with high exposure of polypharmacy. (Table 3)

Table 3: Number of Interactions with Corticosteroids per Prescription

\begin{tabular}{|c|c|c|}
\hline $\begin{array}{c}\text { Number of } \\
\text { drug-drug } \\
\text { Interaction }\end{array}$ & $\begin{array}{c}\text { No. of } \\
\text { Prescription }\end{array}$ & Percentages (\%) \\
\hline 1 & 75 & 67.6 \\
\hline 2 & 28 & 25.3 \\
\hline 3 & 5 & 4.5 \\
\hline 4 & 3 & 2.7 \\
\hline
\end{tabular}

\section{Level/Severity of Drug-Drug Interactions}

The identified drug interactions with corticosteroids were categorized into different levels according to severity, scientific evidence and onset. Among the 154 drug-drug interaction identified, most were of moderate $124(80.5 \%)$ followed by major $21(13.6 \%)$ and contraindicated severity $9(5.8 \%)$. Based on scientific evidence, 75(48.7\%) were good documented, $62(40.3 \%)$ were fair and $17(11 \%)$ were excellent. On assessing the onset of the potential drug-drug interaction, $74(48 \%)$ were with delayed onset, $43(27.9 \%)$ were unspecified and $37(24 \%)$ rapid onset.

The current study showed that, delayed onset was contributed of around $48 \%$ of all the identified drugdrug interactions. This takes relatively longer time to occur their harmful effect and it could be due to frequently administered corticosteroids and higher accumulation effect or larger extent of plasma binding. (Table 4)

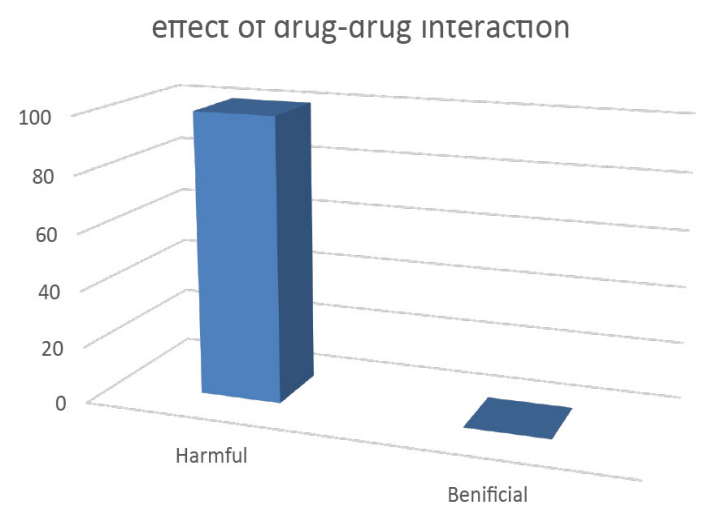

Figure 2: Effect of Drug-Drug Interaction 
Table 4: Level/Severity of Identified Interaction

\begin{tabular}{|c|c|c|}
\hline $\begin{array}{c}\text { Severity of drug- } \\
\text { drug interaction }\end{array}$ & No. of severity & $\begin{array}{c}\text { Percentages } \\
\text { (\%) }\end{array}$ \\
\hline $\begin{array}{c}\text { Severity } \\
\text { Contraindicated }\end{array}$ & 9 & 5.8 \\
Major & 21 & 13.6 \\
Moderate & 124 & 80.5 \\
\hline Documentation & & \\
Excellent & 17 & 11.0 \\
Good & 75 & 48.7 \\
Fair & 62 & 40.3 \\
\hline Onset & & \\
Rapid & 37 & 24.0 \\
Delayed & 74 & 48.0 \\
Unspecified & 43 & 27.1 \\
\hline
\end{tabular}

\section{Common Interaction Drugs}

Table 5 showed common interacting drugs with corticosteroids. Dexamethasone with ciprofloxacin, moxifloxacin, diclofenac, pantoprazole, theophylline and hydrocortisone with diclofenac, phenytoin, theophylline, amlodipine, telmisartan, were the most encountered combinations.

Table 5: Commonly Involved Drug Classes in Drug Interaction

\begin{tabular}{|l|l|}
\hline Classes of Drug & Examples of drug \\
\hline Corticosteroids & Dexamethasone, Prednisolone, Hydrocortisone, Deflazacort, Budesonide \\
\hline Antihypertensive & Amlodipine, Telmisartan, Ramipril, Fosinopril, Hydrochlorothiazide \\
\hline Antibiotics & Ciprofloxacin, Moxifloxacin, Levofloxacin \\
\hline Antimalarial & Artemether \\
\hline Acid Suppressants & Pantoprazole \\
\hline Analgesics & Diclofenac, Mefenamic Acid, Tramadol \\
\hline Others & INH, MTX, Alprazolam, Rifampicin \\
\hline
\end{tabular}

\section{CONCLUSION}

A high prevalence of polypharmacy and drug interactions with corticosteroids was identified in our study. Most of interactions were found to be harmful and may cause tendon rupture, gastroenterology related problems, negative effects on blood pressure and most of were delayed onset in nature. Since the irreversible adverse effects of corticosteroids can be potentiated by use of other concurrent drugs, a regular therapeutic intervention is necessary.

Polypharmacy found in the prescriptions accepted can be accepted, as they are clinically appropriate and/or meet the need of the patient condition, but monitoring is required for the occurrence of drug related problems. Hence, the Clinical Pharmacist act as a potential role in health care system in assisting physician in altering the number of medications taken, the number of doses taken, improving the patient medication adherence, preventing the adverse drug reactions, drug-drug interactions, improve the health related quality of life and decreasing the health care cost of the patient.

\section{ACKNOWLEDGEMENT}

We are grateful to Dr. H. Doddayya, Principal, N.E.T. Pharmacy College, Raichur, Mr. Binu KM, HOD of Department of Pharmacy Practice, Navodaya Medical College \& Research Centre, Raichur, Karnataka, India. 


\section{REFERENCES}

1. John NN, Udupi RH, Binu KM. Incidence of Polypharmacy Induced Drug Interaction in a Tertiary Care Hospital. Int J Pharm Sci Res 2012;3(7):2119-2121.

2. Kumara Swamy RC et al. Prevalence of Polypharmacy and Drug to Drug Interactions in a Tertiary Care Teaching Hospital. Int Res J Pharm 2014;5(10):778-782.

3. Igbiks T, Joseph OF. Drug Prescription Pattern in Nigerian Tertiary Hospital. Tropical Journal of Pharmaceutical Research 2012;11(1):146-152.

4. Rollason V. Reduction of polypharmacy in the elderly: systematic review of the role of pharmacist. Drugs Aging 2003;20:817-832.

5. Linda R Tulner et al. Discrepancies in Reported Drug Use in Geriatric Out-patients: Relevence to Adverse Events and Drug-Drug Interactions. The American Journal of Pharmacotherapy 2009;7(2):93-104.

6. Mahesh Kumar VP, Dhanpal CK. Prevalence of Polypharmacy in Geriatric Patients in
Rural Teaching Hospital. American Journal of Phytomedicine and Clinical Therapeutics 2014;2(3):413-419.

7. David Lee R. Polypharmacy: A case report and new protocol for management. J Am Board Fam Pract 1998;11(2):140-144.

8. Thomas HF, Sweetnam PM, Janchawee B, Luscombe DK. Polypharmacy among older men in South Wales. Eur J Clin Pharmacol 1999;55:411-415.

9. Colley CA, Lucas LM. Polypharmacy: The cure becomes the disease. J Gen Intern Med 1993;8(5):278-283.

10. Ashok Kumar Malpani, Riyaz Miya. Polypharmacy Induced Drug-Drug Interactions at Tertiary Care Teaching Hospital in North Karnataka. World Journal of Pharmaceutical Research 2015;4(7):896-903.

11. Haftay BM, Kalid S. Prevalence of Potential DrugDrug Interactions among Psychiatric Patients in Ayder Referral Hospital, Mekelle, Tigray, Ethiopia. Journal of Scientific \& Innovative Research 2015;4(2):71-75. 